Service social

\title{
La retraite au féminin, par Philippe Pitaud, Paris, Pierre Horay, 1983, 221 pages.
}

\section{Nicolas Zay}

Volume 34, numéro 1, 1985

Personnes âgées, milieux de vie et pratiques sociales

URI : https://id.erudit.org/iderudit/706262ar

DOI : https://doi.org/10.7202/706262ar

Aller au sommaire du numéro

Éditeur(s)

École de service social de l'Université Laval

ISSN

1708-1734 (numérique)

Découvrir la revue

Citer ce compte rendu

Zay, N. (1985). Compte rendu de [La retraite au féminin, par Philippe Pitaud, Paris, Pierre Horay, 1983, 221 pages.] Service social, 34(1), 188-189.

https://doi.org/10.7202/706262ar d'utilisation que vous pouvez consulter en ligne.

https://apropos.erudit.org/fr/usagers/politique-dutilisation/ 
(personnel de soin, bénévoles, etc.) ainsi qu'avec les réseaux sociaux informels et les ressources formelles de la communauté. On y rappelle que l'intervention sociale auprès de la personne âgée et de la famille doit commencer tôt, c'està-dire dès le moment où on effectue des démarches en vue d'un placement.

Le dernier chapitre présente les principales difficultés de l'intervention sociale, qui proviendraient de l'état des connaissances sur le vieillissement et sur l'intervention sociale préventive, des politiques gouvernementales, des procédures des organismes, et des praticiens eux-mêmes. Les auteurs insistent toutefois pour dire que ces difficultés ne sont pas insurmontables mais qu'il est important de les connaître pour les prévenir ou les contourner. Ce chapitre contient aussi des indications sur les attitudes que peuvent prendre les travailleurs sociaux et sur les rôles qu'ils peuvent jouer afin de provoquer des changements et d'améliorer la qualité de l'intervention psychosociale auprès des personnes âgées.

Les auteurs précisent que ce volume s'adresse aux professeurs et aux étudiants impliqués dans des programmes de formation ainsi qu'aux intervenants qui travaillent directement auprès des personnes âgées. Ils ajoutent que ce livre peut également être utile aux administrateurs, planificateurs et superviseurs qui souhaitent augmenter leurs connaissances et leurs habiletés en intervention préventive. Les professionnels des sciences infirmières, de la médecine et de la psychiatrie peuvent également en tirer profit.

Dans l'ensemble, les sujets sont traités avec clarté. Le fait que chaque chapitre soit abondamment illustré d'exemples concrets contribue à l'originalité et à l'intérêt du volume, en plus d'en faciliter la compréhension; il s'agit habituellement d'histoires de cas et de plans d'intervention, souvent même accompagnés de questions adressées au lecteur, de nature à guider la réflexion ou la discussion.

Les auteurs s'attardent davantage aux aspects cliniques de l'intervention ; on retrouve toutefois, à diverses reprises, des suggestions d'intervention de type communautaire. II m'apparait important que cette dimension soit davantage mise en lumière, car le travail avec la communauté, avec ses réseaux formels et informels, me semble indispensable au succès de toute intervention préventive, qu'elle soit primaire, secondaire ou tertiaire.

École de service social,

Lise Darveau-Fournier

Université Laval.

La retraite au féminin, par Philippe PITAUD, Paris, Pierre Horay, 1983, 221 pages.

Jusqu'à très récemment, la retraite était perçue comme un phénomène masculin, même si le travail de la femme hors du foyer n'est pas réellement quelque chose de nouveau. Pendant longtemps c'est donc dans le cadre du passage de l'homme à la retraite qu'on a examiné cette condition. 
L'étude de Pitaud s'inscrit dans une tendance relativement nouvelle. Au lieu de placer l'homme au point central, on y suit la travailleuse dans son cheminement vers sa propre retraite et son insertion dans cette nouvelle étape de vie.

Le premier chapitre est consacré à quelques aspects théoriques et aux grandes caractéristiques sociales qui marquent la femme à l'âge de la retraite. Pour elle, la cessation de l'activité professionnelle signifie un retour à la maison, à la dépendance, et un renforcement des rôles traditionnels. Si elle ressent la rupture moins fortement que l'homme, c'est peut-être parce que le milieu domestico-familial constituait le pôle fort de sa vie, même au temps de la vie active. Par ailleurs, la fin de l'activité professionnelle peut être accentuée, chez elle, par la dévalorisation sociale qu'entraîne son vieillissement.

Vient ensuite un tableau de l'évolution du travail féminin, caractérisé par la discontinuité, c'est-à-dire par des sorties et des retours au marché du travail selon l'évolution du cycle de vie familiale. Cette discontinuité explique pourquoi les plans de pension qui font un lien entre la durée de la vie active et le montant des prestations désavantagent les femmes, dont la majorité ne peut accéder à la pension maximale faute d'avoir travaillé le nombre d'années requis.

Les travailleuses, comme d'ailleurs les travailleurs, font face à un appauvrissement brutal de leur vie relationnelle au moment de cette transition. Elles peuvent se tourner alors vers leur conjoint, leur famille ou s'insérer dans des relations extérieures au cercle familial. Chacune de ces situations est examinée à partir du témoignage des intéressées. La façon dont elles se meuvent dans l'un ou l'autre de ces cadres détermine leurs activités et leurs loisirs.

Pour terminer, disons que l'ouvrage de Philippe Pitaud devrait intéresser tous ceux qui se préoccupent de l'évolution du rôle de la femme dans le monde contemporain. Parce que sa longévité est supérieure à celle de l'homme, elle passera une portion plus longue de son cycle de vie dans la phase qu'on appelle "la vieillesse" et dont les frontières tendent à reculer.

Parmi les conclusions de l'auteur, on peut retenir l'écart qu'il observe entre le changement social, tel qu'il figure dans ce qu'il est convenu d'appeler, en France, le "discours", et les mentalités qui régissent les attitudes et comportements des gens dans la vie de tous les jours. II est difficile de comprendre, cependant, pourquoi il oppose "discours" à "mentalité " puisque ces deux variables font assez bon ménage si l'on se place dans une perspective gérontologique. Si le discours représente un phénomène ponctuel dans le temps, la mentalité est le résultat d'une incorporation progressive des « discours successifs" qui ont accompagné le cours de l'existence humaine.

Que la femme assimile les images du vieillissement féminin que lui renvoie la société ne fait pas de doute. II reste cependant à savoir si elle conserve, en vieillissant, les stéréotypes qui ont eu cours durant la première moitié de sa vie ou si elle les abandonne en faveur des nouveaux stéréotypes prévalant au moment où elle s'apprête à abandonner la vie active. Cette question reste, pour le moment, sans réponse.

École de service social,

Nicolas ZAY Université Laval. 